\title{
Using Mathematical Software in High School Math Class: A Case Study
}

\author{
Hui Xu
}

\begin{abstract}
Mathematical software can be used as valuable teaching tools in high school math education. The software can convey complex math concepts through the use of interactive models and engaging 2D/3D visualizations, making difficult topics come alive and interesting. Maple is advanced mathematical software with powerful symbolic deduction, numerical calculation and graphic display abilities. It is very suitable for high school math class teaching. In this paper, the Newton iteration method for solving single variable nonlinear equation was taken as an example to demonstrate how to introduce math concepts, derive formulae, teach visually and explore deeply by using Maple in the high school math class. The teaching effect was evaluated by comparing the innovative Maple-aid-teaching method with the traditional multimedia teaching mode. It is found that the innovative method achieved better teaching effect.
\end{abstract}

Index Terms-High school math education, mathematical software, maple, Newton iteration method.

\section{INTRODUCTION}

Math has long been very abstract and difficult for high school students. It is now generally accepted that the key point of math education in high school should be focused on training students' creative thought ability rather than imparting basic math knowledge to them. Thus, in math class, teachers should not only explain the details of math concepts and theories, but also should guide the students to observe and think things in the mathematical point of view, help them learn to question, guess and explore in the mathematical world.

With the fast development of IT technology in the recent years, the idea of using computers in the teaching and learning environment has been accepted. However, mathematical software play a relatively minor, somewhat untrusted role in high school math class for achieving the learning objectives, even though they have been widely used in many science and engineering fields for a long time. Nevertheless, some researchers have been working in this direction and have shown that a math teacher with no specialized programming knowledge can use mathematical software as new teaching tools. G. Smerage discussed applications of math software in engineering courses [1]. T. M. Wasfy used a web-based software system named LEA (Learning Environments Agent) for advanced multimedia and virtual-reality education and training [2]. L. M. Gabriel and L. Gilberto reported an

Manuscript received June 3, 2015; revised August 11, 2015.

Hui $\mathrm{Xu}$ is with No. 1 High School attached to Central China Normal University, the No. 1 Special, Tang Xun Hu North Road, Eastlake High and New Technology Development Zone, Wuhan City, Hubei Province, China (e-mail: xuhui_hsy@163.com). electronic collaborative learning environment for motivating students towards mathematics [3]. T. Kabaca et al. used algebra software in teaching and learning mathematical concepts in calculus courses [4]. Y. Phillips discussed the use of graphing calculators, the internet and computer math software in mathematics education [5]. M. A. Abánades et al. mixed geometric software GeoGebra and a free open source algebra system, Sage, to enhance their mathematics education [6]. V. Thakkar et al. created an interactive environment by interfacing 3D animation software and gesture hardware [7]. F. Ji et al. summarized the current status of computer application in mathematics education in primary schools in both China and other countries [8]. K. R. Grimes provided a narrative of work in progress to validate a math app designed for number sense [9].

In the math textbook for high school in Chinese Mainland, the Newton iteration method was introduced to students for solving single variable nonlinear equation [10]. Although they have learned the solution method for linear equation before, the idea of iteration and approximation for solving nonlinear equation are completely new for them. Thus, it is the main task to introduce the iteration and approximation concepts to the students in class. From the author's teaching practice, it is found that students will be confused if the abstract Newton iteration equations are brought to them directly. They cannot picture the iteration process clearly in mind, and they may doubt whether this "repetition of calculation" will give any meaningful results. However, this situation can be changed if teachers use mathematical software as a teaching assistance in class. With the help the mathematical software, teachers can not only help students build up intuitive concepts of iteration and approximation, but also penetrate the thought of combining numbers with pictures. Once the dynamic process of Newton iteration method is distinctly demonstrated step by step in a graphical way, students can get a better grasp of the nature of the method. Moreover, with the help of mathematical software, students can do math experiments instantly and interactively in the class to explore the method on their own, verifying their thought, finding the merits and disadvantages of the method.

There are now many mathematical software systems suitable for math education in high school, such as Maple, Mathematica, MATLAB and ActiveMath. Maple is a widely used powerful system with rich symbol deduction, numerical calculation, and graphical display abilities [11]. As there are many built-in mathematical and graphical functions in Maple, it is relatively simple to write a computation program instinctively. Thus it is very suitable for prompt classroom demonstration.

In this paper, the Newton iteration method for single 
variable nonlinear equation is taken as an example to illustrate how to use Maple in high school math education. The procedure of introducing the concept of approximation by replacing curve with straight line, displaying the iteration process graphically and exploring the method by math experiments will be elaborated in detail. The innovation teaching method was compared with the traditional teaching method by evaluating classroom teaching efficiency, student assessment, homework, and an 8 week later quiz. The results show that utilizing mathematical software in math class teaching is greatly beneficial to high school math education.

\section{TEACHING THE NeWton ITERATION METHOD WITH MAPLE}

\section{A. Single-Variable Fibonacci Cubic Equation}

We start the lesson with a fascinating little history story. In 1225, the famous Italy mathematician Leonardo Fibonacci studied the following single-variable cubic equation which was given as a mathematics competition problem:

$$
f(x):=x^{3}+2 x^{2}+10 x-20=0
$$

Fibonacci proved that the roots of the equation cannot be obtained geometrically by rulers and compasses, and gave an approximate root of 1.3688081078 [12]. What is interesting is that no one knows how he managed to get this remarkable root.

The curve of the function $f(x)$ can be plotted by Maple as shown in Fig. 1. The zero point or the root of the Fibonacci equation can be solved at any precision by Maple, for example, with 20 digits precision the root is 1.3688081078213726352. The Maple code to plot and solve the equation is shown in Fig. 2

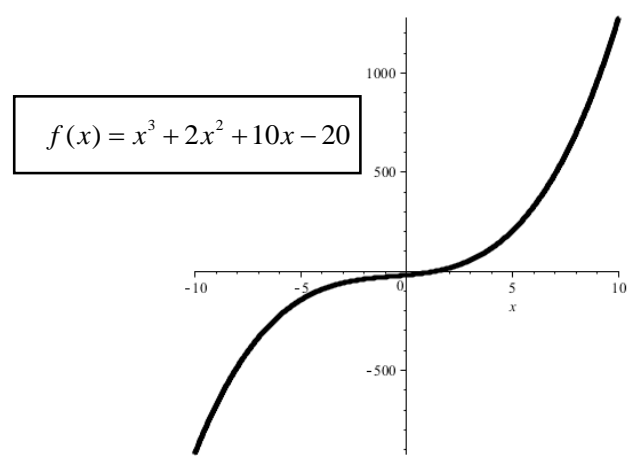

Fig. 1. Curve of the cubic function.

restart $:$ with (plots) : Digits $:=20$ :

fun $:=x^{3}+2 \cdot x^{2}+10 \cdot x-20$

$x$ Start $:=-10: x$ End $:=10$ :

plot (fun, $x=x$ Start ...xEnd $)$;

evalf (solve (fun, $x)$ );

Fig. 2. Maple code to plot and solve the equation.

\section{B. Derivation of the Newton Iteration Method for Single-Variable Equation}

We now investigate the numerical algorithm to solve Fibonacci equation by using first order derivative. It is obvious that function $f(x)$ is first order derivable everywhere. This indicates that there is a unique gradient thus unique tangent for any point on the curve. In order to find the zero point of this cubic function, we use the approximation idea of replacing a curve by a straight line. We take the tangent of the curve at any point as a good approximation of the curve in the vicinity of that point. Thus the zero point of the tangent can be a good guess of the zero point of the curve. Let $f^{\prime}\left(x_{0}\right)$ be the slope of $f(x)$ at point $\left(x_{0}, f\left(x_{0}\right)\right)$, the expression of tangent is $y=f\left(x_{0}\right)+f^{\prime}\left(x_{0}\right)\left(x-x_{0}\right)$. When $f^{\prime}\left(x_{0}\right) \neq 0$, the zero point of above tangent equation can be easily solved to be $x=x_{0}-f\left(x_{0}\right) / f^{\prime}\left(x_{0}\right)$. Suppose that we have done $(n-1)$ th calculation and got an approximate root $x_{n-1}$, we can modify this approximate root to evaluate a better root $x_{n}$ :

$$
x_{n}=x_{n-1}-\frac{f\left(x_{n-1}\right)}{f^{\prime}\left(x_{n-1}\right)} \text {, if } f^{\prime}\left(x_{n-1}\right) \neq 0
$$

Thus from a given initial guessed root $x_{0}$ of the equation, a serial approximate roots $x_{i}, i=0,1,2, \ldots$ can be obtained. This forms an iteration process. The iteration process stops once the root satisfies the given precision. There are two ways to test the convergence of the iteration. One is to test whether $f\left(x_{n}\right)=0$. Notice that this cannot be satisfied strictly, we use $\left|f\left(x_{n}\right)\right| \leq \varepsilon$ in numerical algorithm instead, where $\varepsilon$ is small positive real. The second criterion is to check the relative error of two consequent roots, $\left|\left(x_{n}-x_{n-1}\right) / x_{n-1}\right| \leq z_{0}$, where $z_{0}$ is some small positive factor. It can be easily proved that the two criterions are equal and we use the second criterion in our Maple code:

$$
\begin{aligned}
& x_{n}=x_{n-1}-\frac{f\left(x_{n-1}\right)}{f^{\prime}\left(x_{n-1}\right)} \Rightarrow x_{n}-x_{n-1}=-\frac{f\left(x_{n-1}\right)}{f^{\prime}\left(x_{n-1}\right)} \\
& \Rightarrow\left|\frac{x_{n}-x_{n-1}}{x_{n-1}}\right|=\left|\frac{f\left(x_{n-1}\right)}{f^{\prime}\left(x_{n-1}\right) x_{n-1}}\right| \\
& \Rightarrow\left|\frac{x_{n}-x_{n-1}}{x_{n-1}}\right| \leq\left|\frac{\varepsilon}{f^{\prime}\left(x_{n-1}\right) x_{n-1}}\right|=z_{0}
\end{aligned}
$$

\section{Coding and Graphical Presentation of the Newton Iteration Procedure}

The flow chart of the Newton algorithm is given in the textbook as a guide for students to write a computer program. However, high school students in Chinese Mainland have weak coding skills and it is really a tough work for them to write computer program with high-level programming languages, such as Basic, $\mathrm{C}$ and Fortran. Moreover, it is beyond their abilities to write a postprocessor to graphically illustrate the calculation process. So the high-level programming languages are not suitable for class performance in high school. We use Maple software in our classroom to show how to code the algorithm, to reveal the 
details of iteration process of Newton method, and finally display the process graphically and interactively. Take Fibonacci equation as an example, the initial guessed root is 4, and the error limit is $10^{-9}$. Maple code for Newton iteration method is listed in Fig. 3. The iteration process is graphically shown in Fig. 4. The approximate root sequence produced by the code is

$\{4,2.4324324324324324324,1.6173339583980784526$, $1.3855811608523389600, \quad 1.3688891989982056470$,

$1.3688081097247388091,1.3688081078213726363\}$.

It can be seen that we get the result of Fibonacci's within seven iterations.

Although the algorithm of Newton iteration method is simple and can be easily understood by students, there are still some issues behind that confusing them. For example, students in my class wondered that: Can the iteration process of Newton method be carried on ceaselessly before reaching a root satisfying the error limit? Should the approximate root produced by Newton method approach infinitely to the exact root of the equation? What factors influence the convergence of the iteration? With the help of Maple, the students in my class find the answers themselves by exploring these issues through math experiments which will be addressed in next section.

\section{EXPLORING THE NEWTON ITERATION METHOD BY DOING MATH EXPERIMENTS IN THE MAPLE SYSTEM}

\section{A. Math Experiment 1: Iteration Is Disrupted When the} Zero First Order Derivative Point Is Met

Students can immediately find that the iteration process cannot execute if $f^{\prime}\left(x_{k}\right)=0$. We take equation $x^{3}-5 x=0$ as an example and do math experiments. When we choose initial guess $x_{0}= \pm \sqrt{5 / 3}$, the first order derivative of $f(x)=x^{3}-5 x$ is zero at $x_{0}$, and the tangent of function at that point is horizontal. Thus the tangent will never meet the $\mathrm{x}$ axis, and there will be no zero point and the iteration cannot continue, as shown in Fig. 5.

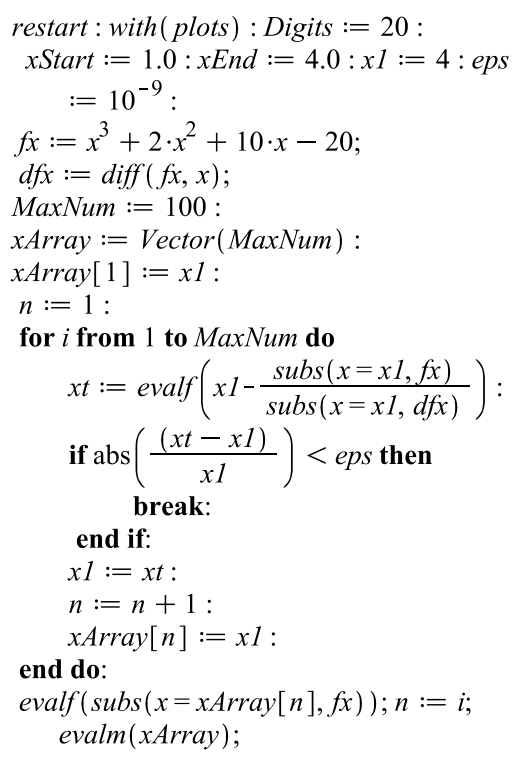

Fig. 3(a). Maple code for Newton iteration method.

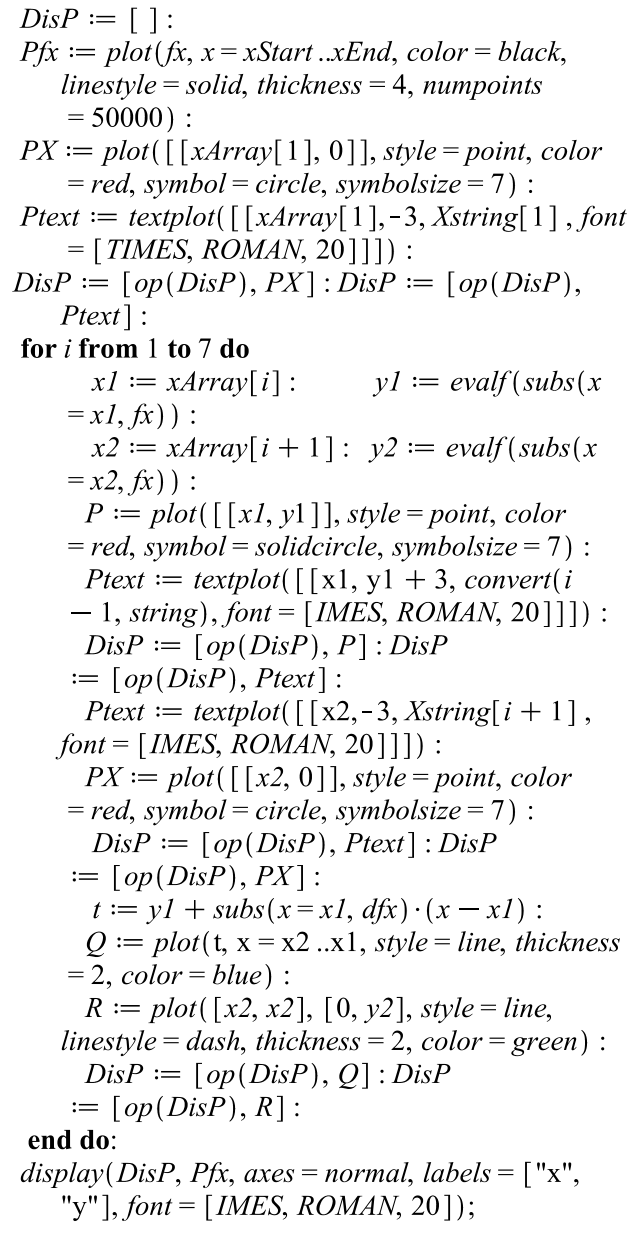

Fig. 3(b). Maple code for display.

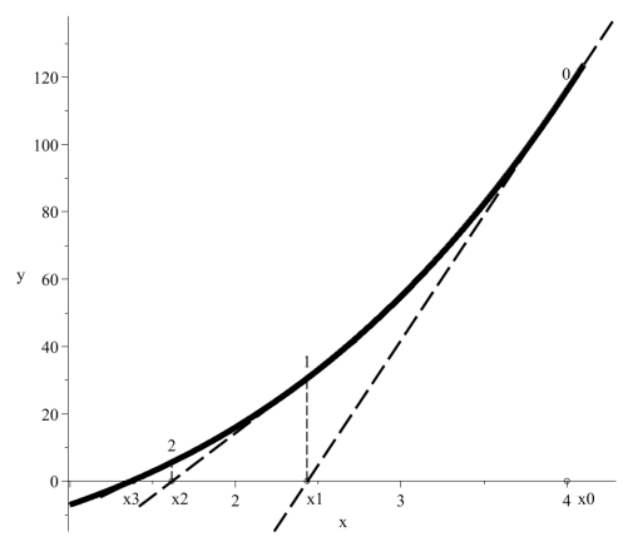

Fig. 4. Solving the Fibonacci equation by Newton iteration method.

Going a step further, the students can imagine that if the slope at any points be zero in the iteration process, the Newton iteration cannot continue. This can be easily tested by Maple. Suppose that we met the critical point in our second iteration, i.e. $f^{\prime}\left(x_{1}\right)=0$, or $x_{1}= \pm \sqrt{5 / 3}$. The value of $x_{0}$ can be obtained from the following equation by the built-in function solve( ):

$$
x_{1}=x_{0}-\frac{x_{0}^{3}-5 x_{0}}{3 x_{0}^{2}-5}= \pm \sqrt{\frac{5}{3}}
$$

Let $x_{0}$ be the real root of above equation, and execute the Newton iteration code in Maple, it can be found that the method fails in the second iteration. 


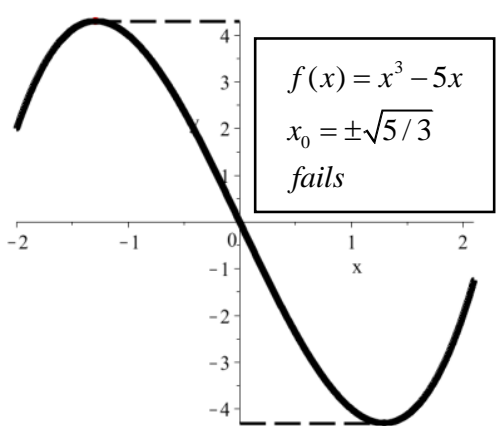

Fig. 5. First order derivative of $f(x)=x^{3}-5 x$ at $x_{0}= \pm \sqrt{5 / 3}$ is zero.

\section{B. Math Experiment 2: Different Initial Guess Leads to Different Results}

We do our math experiments with equation $x^{3}-5 x=0$ again. If we choose $x_{0}=-4$, then we get a convergent root $x=-2.236$ after 6 iterations, as shown in Fig. 6(a). If we choose $x_{0}=0.8$, then we get a convergent root $x=0$ after 4 iterations, as shown in Fig. 6(b). If we choose $x_{0}=1.5$, then we get a convergent root $x=2.236$ after 7 iterations, as shown in Fig. 6(c). This clearly indicates that when using Newton iteration method, different initial guess may lead to different roots.
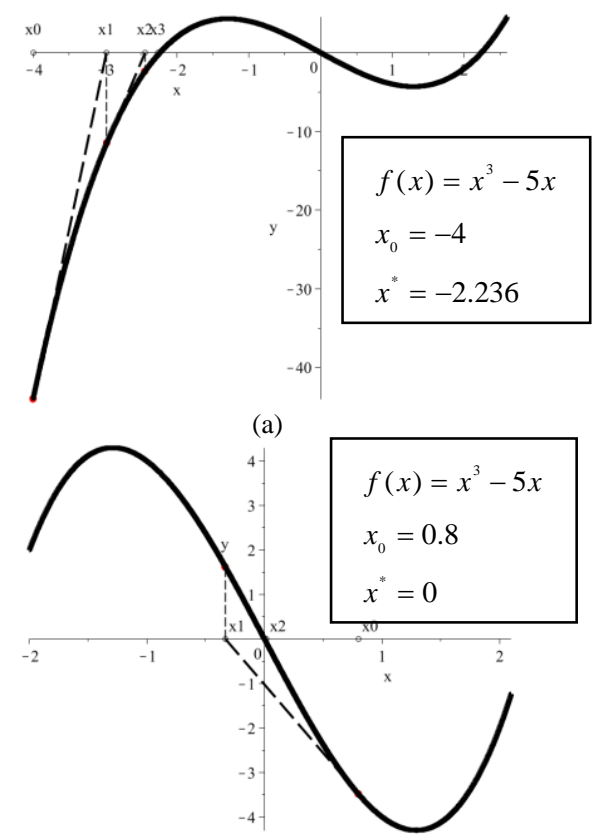

(b)

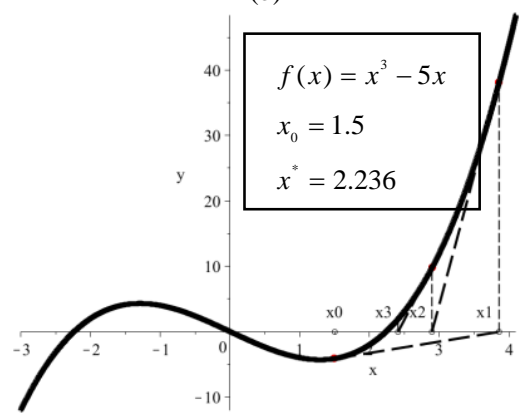

(c)

Fig. 6. Solving $x^{3}-5 x=0$ by Newton iteration method with initial guess (a) $x_{0}=-4$, (b) $x_{0}=0.8$, (c) $x_{0}=1.5$.

\section{Math Experiment 3: The Method Fails because the Iteration Diverges}

Let examine equation $x^{1 / 3}=0$ by numerical experiments. It can be found that whatever $x_{0} \neq 0$ we choose as an initial guess of the root, the following approximate root goes bigger and bigger, i.e. the iteration diverges. Fig. 7 shows several iteration steps with $x_{0}=1$. From this numerical experiment, the students can conclude that for some equations the method may diverge with any initial points.

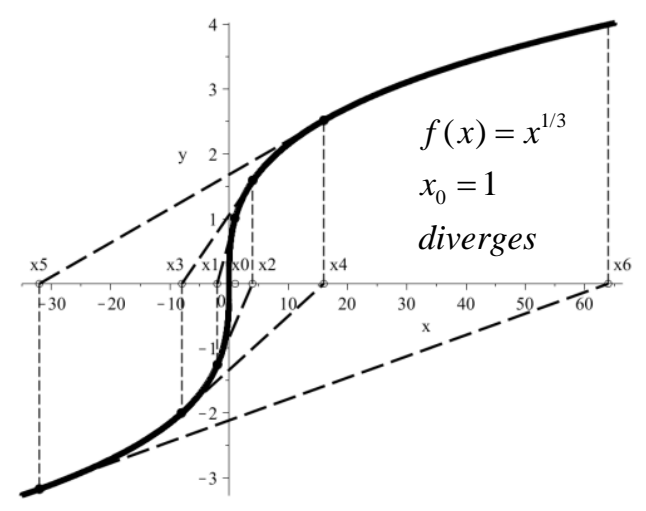

Fig. 7. Newton iteration method diverges in solving $x^{1 / 3}=0$.

\section{Math Experiment 4: The Method Fails Because the Iteration Oscillates}

Can Newton iteration always go on once the zero first order derivative cases have been excluded? Let's do math experiments with equation $x^{3}-5 x=0$. When we take $x_{0}=1$, it is found that the iteration jumps back and forth between two points $x=-1$ and $x=1$, thus the method fails, as shown in Fig. 8(a). A similar example is equation $-x^{3}+6 x^{2}-10 x+6=0$ with $x_{0}=2$. The Newton iteration oscillates between points $x=1$ and $x=2$, as shown in Fig. 8(b). Equation $x^{3}-2 x+2=0$ is another example of this kind. When we take $x_{0}=0.1$, the iterations oscillates between $x=0$ and $x=1$ after several iterations, as shown in Fig. 8(c). For equation $x^{3}-x-3=0$ with $x_{0}=0$, the iteration oscillates between four points $x=-3.0005$, $x=-1.9619, x=-1.1475$, and $x=-0.0074$, as shown in Fig. 8(d). Thus, from these math experiments we found that the Newton method may fail even if the there is no zero tangent point in the iteration. However, if we choose the right initial guess of roots in these experiments, the Newton method will give the right answer.

Through the four math experiments above, students can arrive at following conclusions on Newton iteration method for single variable equation by themselves:

1) From math experiment 1 , it can be concluded that if the first order derivative of the function at any iteration point is zero, the method may fail.

2) From math experiment 2, it can be concluded that different initial guess may converge to different roots.

3) From math experiment 3 , it can be concluded that if the first order derivative of $f(x)$ does not exist in the 
neighborhood of root, the method may fail because of divergence.

4) From math experiment 4, it can be concluded that for some initial guesses of root, the method may fail because of oscillation.

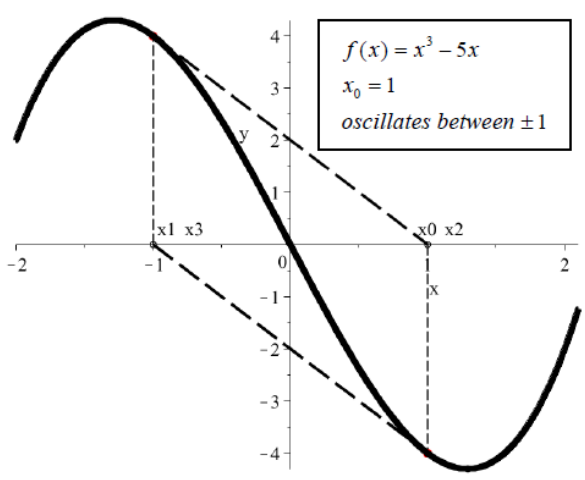

Fig. 8(a). $x^{3}-5 x=0, \quad x_{0}=1$.

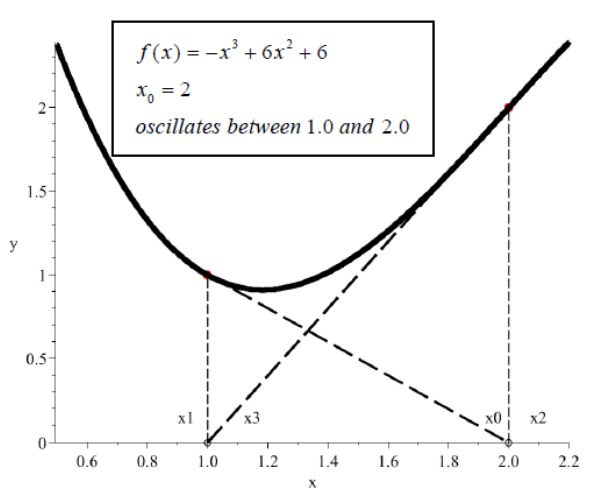

Fig. 8(b). $-x^{3}+6 x^{2}-10 x+6=0, \quad x_{0}=2$.

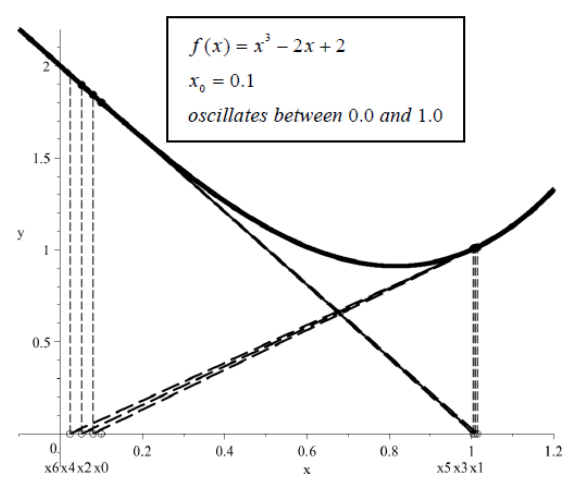

Fig. 8(c). $x^{3}-2 x+2=0, \quad x_{0}=0.1$.

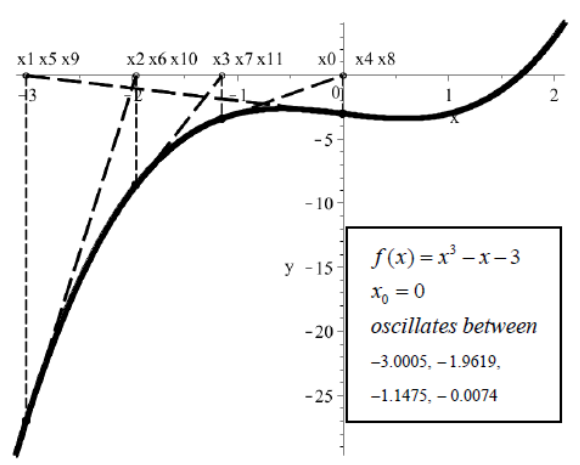

Fig. 8(d). $x^{3}-x-3=0, x_{0}=0$.

Fig. 8. Newton iteration method fails due to oscillation.

\section{TEACHING EFFECT EVALUATION}

We evaluate the teaching effect of using Maple in math class by comparing two parallel classes in our school. One class has 51 students and was taught by the innovate method using Maple, the other class has 53 students and was taught by the traditional multimedia method. The teaching efficiency, students' activities in class, students' remarks on the lesson, the scores of the homework immediately after class and scores of a quiz eight weeks after the class were compared. The homework immediately after the class is "Write in details the procedure for solving equation $f(x)=0$ by Newton iteration method". The test questions 8 weeks after the class are shown in Table I. The results of comparison are shown in Table II. The percent of errors in the students' homework on writing the details of Newton iteration procedure is shown in Fig. 9. The data obviously support the fact that the innovative teaching method using maple is more advantageous than the traditional method.

TABLE I: TEST QUESTIONS 8 WEEKS AFTER THE CLASS

\begin{tabular}{c|l|c}
\hline No. & \multicolumn{1}{|c}{ Questions } & Score \\
\hline 1 & $\begin{array}{l}\text { Describe the procedure of the Newton iteration } \\
\text { method for single variable equation. }\end{array}$ & 50 \\
\hline 2 & $\begin{array}{l}\text { Graphically illustrate what happens on the } \\
\text { Newton method when an iteration point has zero } \\
\text { first order derivative. }\end{array}$ & 15 \\
\hline 3 & $\begin{array}{l}\text { Graphically explain different initial guesses can } \\
\text { lead to different roots. }\end{array}$ & 10 \\
\hline 5 & $\begin{array}{l}\text { Can you graphically illustrate the cases where } \\
\text { Newton iteration method diverges? }\end{array}$ & 10 \\
\hline 5 & $\begin{array}{l}\text { Can you graphically illustrate the cases where } \\
\text { Newton iteration method fails because of } \\
\text { oscillation? }\end{array}$ & \\
\hline
\end{tabular}

TABLE II: RESULTS OF THE COMPARISON (CLASS 1 WAS TAUGHT BY THE INNOVATIVE METHOD, ClASS 2 WAS TAUGHT BY THE TRADITIONAL

\begin{tabular}{|c|c|c|}
\hline \multicolumn{1}{|c|}{ METHOD) } & $\begin{array}{c}\text { Class 1 } \\
\text { (innovative } \\
\text { method) }\end{array}$ & $\begin{array}{c}\text { Class 2 } \\
\text { (traditional } \\
\text { method) }\end{array}$ \\
\hline $\begin{array}{c}\text { Teaching efficiency (time to finish } \\
\text { classroom teaching) }\end{array}$ & 42 minutes & 36 minutes \\
\hline $\begin{array}{c}\text { Students' activities in class (full } \\
\text { mark 100) }\end{array}$ & 95 & 82 \\
\hline $\begin{array}{c}\text { Students' remarks on the lesson } \\
\text { distrustful }\end{array}$ & $\begin{array}{c}\text { Interesting, } \\
\text { dramatic, } \\
\text { beneficial }\end{array}$ & 84 \\
\hline $\begin{array}{c}\text { Average scores of the homework } \\
\text { immediately after the class (full } \\
\text { mark 100) }\end{array}$ & 96 & 43 \\
\hline $\begin{array}{c}\text { Average scores of test questions } 8 \\
\text { weeks after the class }\end{array}$ & 82 & \\
\hline
\end{tabular}

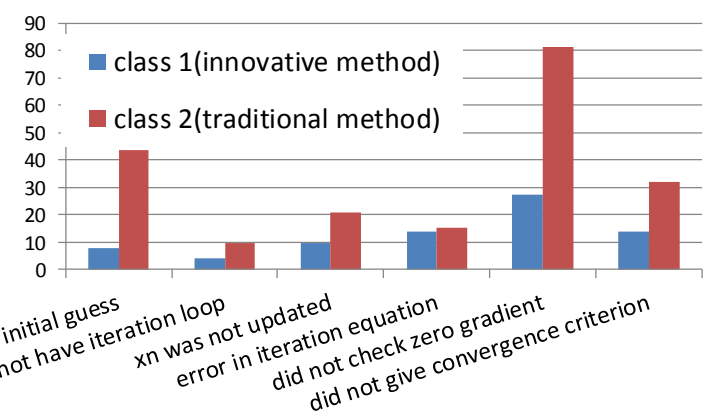

Fig. 9. The percent of errors in the students' homework on writing the details of Newton iteration procedure. 


\section{CONCLUSION}

Mathematical software Maple can be employed as a powerful teaching aid in high school math class. With the help of Maple, the teaching and learning activities can be intuitive, interactive and interesting. Students can understand the algorithm of the method by following teacher's demonstration of the whole procedure with Maple. Students can not only bring out their questions and guesses but also do math experiments by Maple to explore the conditions where the method fails. By evaluating the teaching effect of two classes, one used the innovative method and the other used traditional method, it was found that the innovative method using mathematical software is more favorable and worth popularizing in high school math education.

\section{REFERENCES}

[1] G. Smerage, "Applications of math software in engineering courses," presented at ASEE Annual Conference and Exposition: Vive L'ingenieur, Montreal, Que., Canada, June 16-19, 2002.

[2] T. M. Wasfy, "Lea: Software system for multimedia and virtual-reality web-based education and training," presented at ASME International Design Engineering Technical Conferences and Computers and Information In Engineering Conference, DETC2006, Philadelphia, PA, United States, September 10-13, 2006

[3] L. M. Gabriel and L. Gilberto, "Computer support for learning mathematics: A learning environment based on recreational learning objects," Computers \& Education, vol. 48, pp. 618-641, 2007.

[4] T. Kabaca, Y. Aksoy, M. Aktumen, and S. Mirasyediglu, "The use of computer algebra systems in calculus teaching: principles and sample applications," Technology, Education and Development, Croatia, 2009, pp. 479-500.
[5] Y. Phillips, "The use of technology in mathematics education," presented at 2nd International Conference on Education Technology and Computer (ICETC), Shanghai, China, Jun 22-24, 2010.

[6] M. A. Abánades, F. Botana, J. Escribano, and J. L. Valcarce, "Using free open source software for intelligent geometric computing," presented at 2011 International Conference on Computational Science and Its Applications, ICCSA 2011, Santander, Spain, June 20-21, 2011.

[7] V. Thakkar, A. Shah, M. Thakkar, A. Joshi, and N. Mendjoge, "Learning math using gesture," presented at 2012 International Conference on Education and e-Learning Innovations, Sousse, Tunisia, July 1-3, 2012

[8] F. Ji, H. Zhang, Z. Sun, and K. He, "Developing a cognitive system: An integrated approach of object, algebra and geometry," presented at 6th International Multi-Conference on Society, Cybernetics and Informatics, Orlando, FL, United States, July 17-20, 2012.

[9] K. R. Grimes, "It's just like learning, only fun - A teacher's perspective of empirically validating effectiveness of a math app," presented at 11th International Conference on Cognition and Exploratory Learning in Digital Age, Porto, Portugal, 2014.

[10] S. X. Liu et al, High School Curriculum Standard Textbook: Mathematics, People's Education Press, Beijing, 2013.

[11] Maplesoft. [Online]. Available: http://www.maplesoft.com

[12] D. E. Smith, History of Mathematics, Dover Publications, New York, 1958.

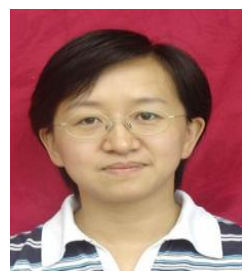

Hui Xu was born on October 20, 1971, in Wuhan city, China. She got her BS of math education in Central China Normal University in 1993. She is now working on the math education in No. 1 High Schoo attached to Central China Normal University. Her current research focuses on the innovative methods in high school math class teaching. She is titled as an academic pacemaker in high school math education of Wuhan city. 